\title{
Efficiency Increase in On-Chip Buck Converter by Introduction of High Permeability Material to Inductor on Interposer H. FUKETA ${ }^{\text {a) }}$, Y. SHINOZUKA ${ }^{\text {a) }}$, K. ISHIDA ${ }^{\text {a) }}$, M. TAKAMIYA ${ }^{\text {a) }}$, T. FUJII ${ }^{\text {b) }}$, H. SHIMIZU ${ }^{\text {b) }}$, K. KOBAYASHI ${ }^{\text {) }}$, T.SATO ${ }^{\text {() }}$, and T. SAKURAI ${ }^{\text {a) }}$
}

a) Institute of Industrial Science, University of Tokyo, 4-6-1 Komaba, Meguro-ku, Tokyo 153-8505, Japan

b) SHINKO Electric Industries Co., Ltd., 36 Kita Owaribe, Nagano-shi, Nagano 381-0014, Japan

c) Shinshu University, 4-17-1 Wakasato, Nagano-shi, Nagano 380-8553, Japan

Abstract

In this paper, an inductor on interposer with a ferrite film is investigated for power supply circuits in order to improve the efficiency of the power supply circuits by introducing a high permeability material to the inductor. A DC-DC buck converter is used as a power supply circuit in this work. The dependence of the inductance on the location of ferrite film is discussed with field electromagnetic simulations. Simulation results indicate that the ferrite film should be located on both upper and lower sides of the inductor and between metals. By using the inductor with the $10 \mu \mathrm{m}$-thick ferrite film, the efficiency of buck converter is improved by $13 \%$ when the efficiency of buck converter with the air core inductor is $60 \%$.

Key Words: Buck Converter, Ferrite, Interposer

\section{INTRODUTION}

Since mobile devices have been currently smaller and thinner, miniaturization of inductors used for power supply circuits is required. Although it is one of promising solutions that inductors are implemented in IC package or interposer, the inductance and Q factor of such inductors are smaller, which results in worsening the efficiency of power supply circuits. Therefore, introduction of high permeability material to the inductor is expected to improve the efficiency. In this paper, the efficiency using inductor on interposer with a ferrite film is investigated as shown in Fig. 1. A DC-DC buck converter is used as a power supply circuit in this work. In order to improve the efficiency of buck converter, the location of ferrite film is also discussed with electromagnetic field simulations.

\section{SIMULATION SETUP}

Fig. 2 shows a circuit schematic of buck converter evaluated in this paper. A controller circuit, buffers, and drivers of buck converter are implemented in the IC, while an inductor is fabricated in the interposer using metal interconnects of the interposer. $\mathrm{V}_{\mathrm{IN}}$ and $\mathrm{V}_{\mathrm{OUT}}$ are input and output voltages of buck converter, respectively.

According to [1], the efficiency $\eta$ of optimally-designed buck converter is expressed as

$$
\eta=\frac{P_{\text {out }}}{P_{\text {out }}+P_{\text {loss }}}=\frac{1}{1+\frac{P_{\text {loss }}}{P_{\text {out }}}}=\frac{1}{1+\frac{1}{D} \sqrt[3]{24 \frac{R_{0} C_{0}}{\tau_{L}} D(1-D)}}
$$

As defined in [1], $\mathrm{P}_{\text {out }}$ and $\mathrm{P}_{\text {loss }}$ represent the output power of buck converter and its power loss, respectively, $\mathrm{R}_{0} \mathrm{C}_{0}$ is the technology-dependent parameter of the IC, and $D$ is a duty ratio, which is given by $\mathrm{V}_{\mathrm{OUT}} / \mathrm{V}_{\mathrm{IN}}$.

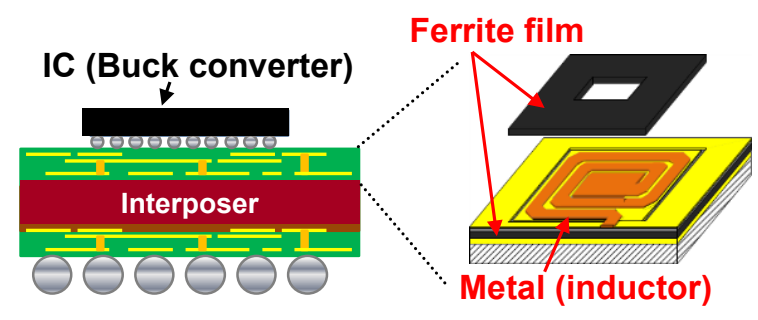

Fig. 1 Inductor on interposer with ferrite film.

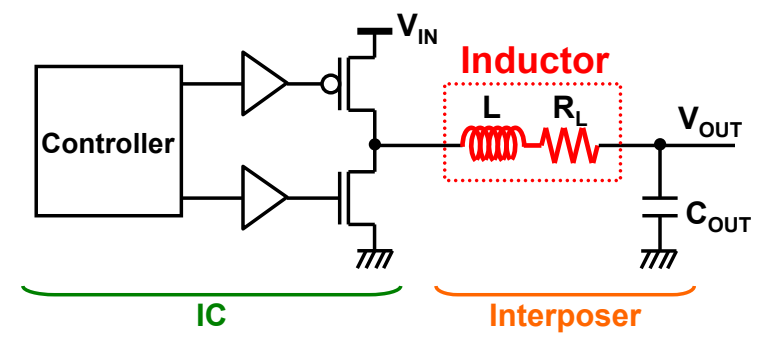

Fig. 2 Circuit schematic of buck converter. 
$\tau_{L}$ in (1) is defined as

$$
\tau_{L}=L / R_{L}
$$

where $L$ and $R_{L}$ are the inductance and the DC resistance of the inductor, respectively. Equations (1) and (2) indicate that large $\tau_{L}$ improves $\eta$ of buck converter and hence $\tau_{L}$ is evaluated in addition to $L$ in this paper.

Fig. 3 shows the layout of the inductor used for simulations described in Section III. Table I summarize the simulation setup. The inductor is simulated by a 3D electromagnetic simulator (Agilent EMPro). In this paper, the dependence of relative permeability on frequency is not considered. The superimposed DC current characteristics are negligible at the output current of $1 \mathrm{~A}$, and hence the characteristics are not considered in this paper.

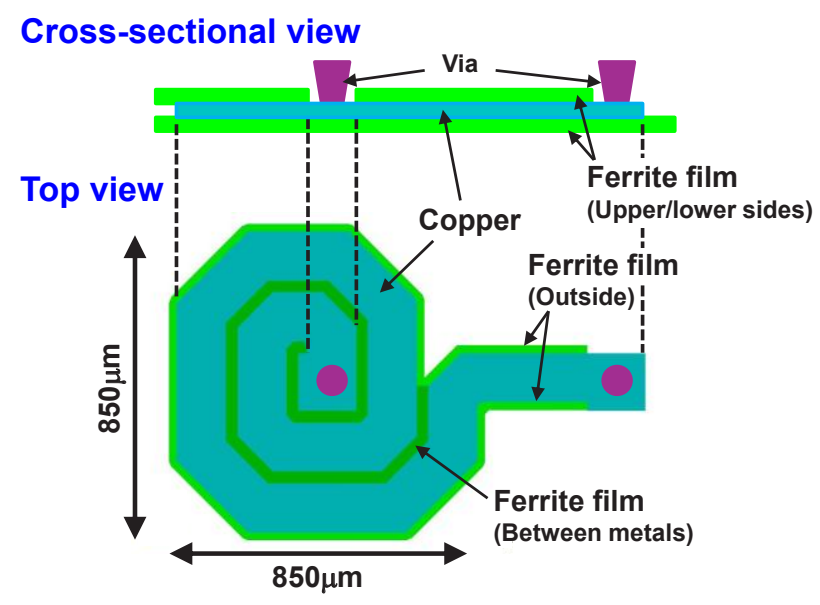

Table I Summary of simulation setup.

\begin{tabular}{|l|l|} 
Inductor \\
\hline Metal & Copper \\
\hline Thickness/width of metal & $20 \mu \mathrm{m} / 130 \mu \mathrm{m}$ \\
\hline Diameter & $850 \mu \mathrm{m}$ \\
\hline Number of turns & 2 \\
\hline Ferrite film & \multicolumn{2}{|l|}{} \\
\hline Relative permeability & 80 \\
\hline Thickness & $1 \mu \mathrm{m}-100 \mu \mathrm{m}$ \\
\hline Buck converter & \multicolumn{2}{|l}{} \\
\hline Input/output voltage & $1.8 \mathrm{~V} / 1.0 \mathrm{~V}$ \\
\hline Output current & $1.0 \mathrm{~A}$ \\
\hline Switching frequency & $10,100 \mathrm{MHz}$ \\
\hline
\end{tabular}

Fig. 3 Layout of inductor used for simulations.

\section{SIMULTATION RESULTS}

\section{A. Dependence of Inductance on Location of Ferrite Film}

In order to investigate the dependence of inductance on the location of the ferrite film, the following two conditions are evaluated; 1) the ferrite film is located on both upper and lower sides of the inductor or only lower side of inductor, and 2) the ferrite film is located between metals or not.

Fig. 4 shows the inductance when the ferrite film is located on both upper and lower sides of the inductor and only lower side of the inductor. The inductance with the ferrite film located on both upper and lower sides of the inductor is much higher than that with the ferrite film located on only lower side of inductor. Fig. 5 illustrates the inductances with and

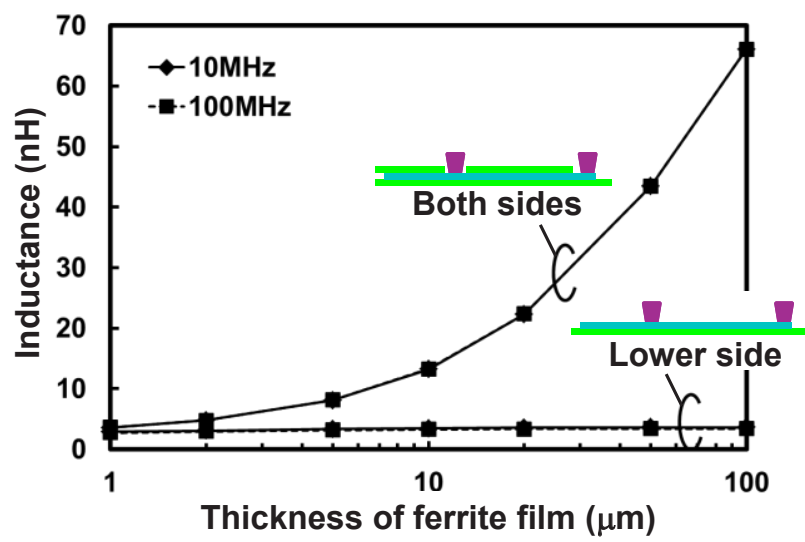

Fig. 4 Inductance when ferrite film is located on both upper and lower sides of inductor and only lower side of inductor.

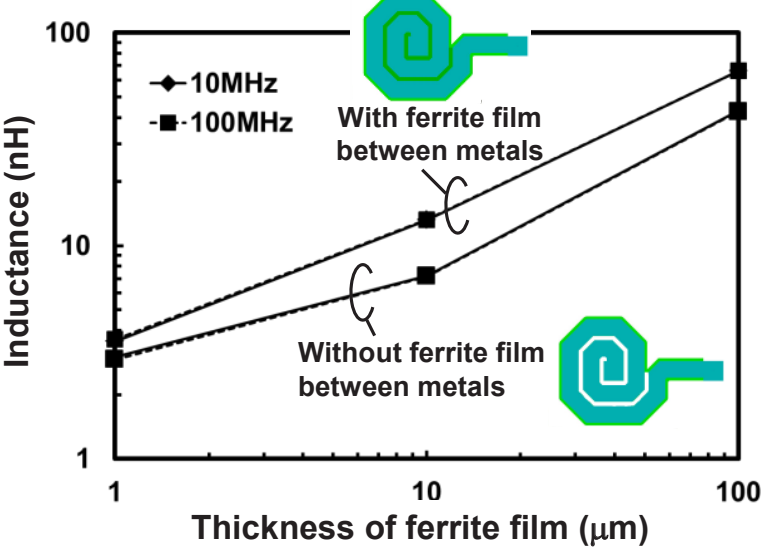

Fig. 5 Inductance with and without ferrite film between metals. 
without the ferrite film between metals (the ferrite film between metals is indicated in Fig. 3). In both cases, the ferrite film is located on the upper and lower sides of the inductor (the thickness of the ferrite film is $10 \mu \mathrm{m}$ ) and the outside of the inductor (the width of ferrite film is $10 \mu \mathrm{m}$ ). The ferrite film between metals improves inductance by $20-60 \%$.

The increase in $\tau_{L}$ improves the efficiency of buck converter as indicated in (1). In order to increase $\tau_{L}$, Figs. 4 and 5 indicate that the ferrite film should be located on both upper and lower sides of the inductor as well as between metals, because $\tau_{L}$ depends on $\mathrm{L}$ as shown in (2).

\section{B. Efficiency Improvement of Buck Converter by Using Inductor with Ferrite Film}

In order to investigate the efficiency of buck converter improved by using the inductor with the ferrite film, $\tau_{L}$, which is derived in (2), is evaluated. Fig. 6 shows the dependence of $\tau_{L}$ on the thickness of the ferrite film. $\tau_{L}$ (ferrite) and $\tau_{L}$ (air) are $\tau_{L}$ of the inductor with the ferrite film and the air core inductor, respectively. When the thickness of the ferrite film is $10 \mu \mathrm{m}, \tau_{L}$ (ferrite) increases by 5.7 times compared with $\tau_{L}$ (air), as indicated in Fig. 6 .

Fig. 7 shows the efficiency improvement of buck converter by using the inductor with the ferrite film compared with the efficiency with the air core inductor when the switching frequency is $100 \mathrm{MHz}$. By using the inductor with the $10 \mu \mathrm{m}$-thick ferrite film, the efficiency of buck converter is improved by $13 \%$ and $8 \%$ when the efficiencies of buck converter with the air core inductor are 60 and $80 \%$, respectively.

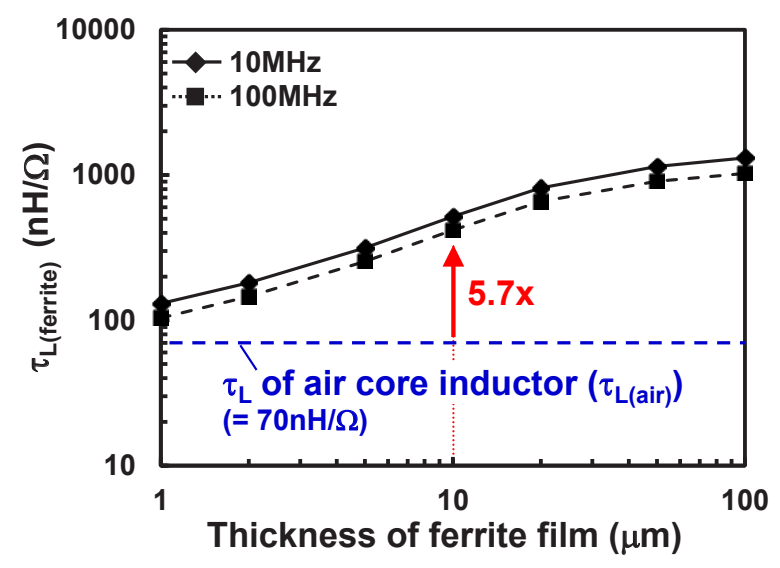

Fig. 6 Dependence of $\tau_{L}$ of inductor with ferrite film and air core inductor.

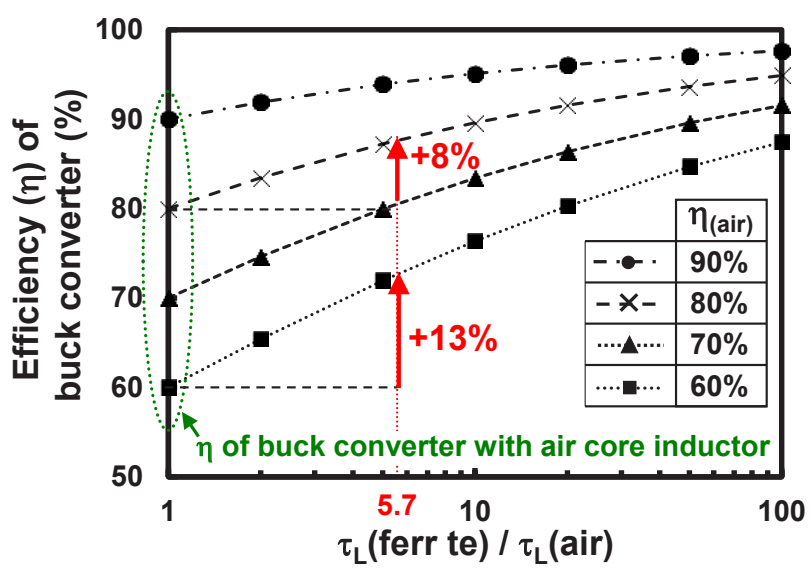

Fig. 7 Efficiency $(\eta)$ improvement of buck converter by using inductor with ferrite film. $\eta_{\text {(air) }}$ is $\eta$ of buck converter with air core inductor.

\section{CONCLUSION}

In this paper, an inductor on interposer with a ferrite film was investigated for power supply circuits. A DC-DC buck converter was used as a power supply circuit in this work. The inductance and $\tau_{L}$ improved by the ferrite film was evaluated with field electromagnetic simulations. First, the dependence of the inductance on the location of the ferrite film was discussed. Simulation results indicate that inductance with the ferrite film located on both upper and lower sides of the inductor is much higher than that with the ferrite film located on only lower side of inductor. Furthermore, the ferrite film between metals improves inductance by $20-60 \%$. These results indicate that the ferrite film should be located on both upper and lower sides of the inductor as well as between metals. Then, the efficiency improvement of buck converter by using the inductor with the ferrite film was evaluated. By using the inductor with the $10 \mu \mathrm{m}$-thick ferrite film, the efficiency of buck converter is improved by $13 \%$ when the efficiency of buck converter with the air core inductor is $60 \%$. An investigation considering the dependence of relative permeability on frequency is future work.

\section{REFERENCES}

[1] G. Schrom, P. Hazucha, F. Paillet, D. S. Gardner, S. T. Moon, and T. Karnik, "Optimal Design of Monolithic Integrated DC-DC Converters," Proc. IEEE International Conference on IC Design and Technology, pp.1-3, 2006. 